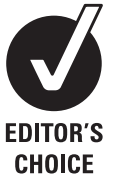

${ }^{1}$ Deakin University—Northern Health Clinical Partnership, School of Nursing, Deakin University, Victoria, Australia ${ }^{2}$ Emergency Department, The Northern Hospital, Northern Health, Victoria, Australia

\section{Correspondence to} Dr Julie Considine, Deakin University-Northern Health Clinical Partnership, School of Nursing, Deakin University, 221 Burwood Hwy, Burwood, Victoria 3125, Australia; julie.considine@deakin.edu.au

Accepted 25 November 2009 Published Online First 26 June 2010

\title{
Effect of clinician designation on emergency department fast track performance
}

\author{
Julie Considine, ${ }^{1}$ Matthew Kropman, ${ }^{2}$ Helen E Stergiou ${ }^{2}$
}

\begin{abstract}
Objective To examine the effect of clinician designation on emergency department (ED) fast track performance.

Design and Setting A retrospective audit of patients managed in the fast track area of an ED in metropolitan Melbourne, Australia.
\end{abstract}

Participants Patients triaged to ED fast track from 1 January 2008 to 31 December 2008 ( $n=8714)$.

Main Outcome Measures Waiting times in relation to Australasian triage scale (ATS) recommendations and ED length of stay (LOS) for non-admitted patients were examined for each clinician group.

Results Compliance with ATS waiting time recommendations was highest (82.5\%) for emergency nurse practitioners/candidates and lowest (48.2\%) for junior medical officers. Median ED LOS was less than $3 \mathrm{~h}$ for non-admitted patients, and $85.8 \%$ of non-admitted fast track patients $(n=6278$ ) left the ED within $4 \mathrm{~h}$. Patients managed by emergency nurse practitioners/ candidates had the shortest ED LOS (median $1.7 \mathrm{~h}$ ) and patients managed by junior medical officers and locum medical officers the longest ED LOS (median $2.7 \mathrm{~h}$ ) $\left(\chi^{2}=498.539, d f=6, p<0.001\right)$.

Conclusions Clinician designation does impact on waiting times and, to a lesser extent, ED LOS for patients managed in ED fast track systems. Future research should focus on obtaining a better understanding of the relationship between clinician expertise, time-based performance measures and quality of care indicators.

Emergency department (ED) fast track systems have evolved to improve the management of patients with non-urgent complaints by decreasing waiting time, ED length of stay (LOS) and overcrowding, and increasing patient and staff satisfaction with ED care. ${ }^{1-6}$ A statewide survey of fast track services in Victorian ED showed most metropolitan and major rural ED in Victoria had a formal fast track system. ${ }^{7}$ The majority of fast track systems in Victorian ED operated during hours that matched periods of peak demand and most ED offered a fast track service for $12-16 \mathrm{~h}$ per day. ${ }^{7}$

In order to optimise the function and capacity of ED fast track systems, a geographically dedicated area staffed by dedicated senior medical and nursing staff is recommended..$^{148}$ It is proposed that dedicated senior medical and nursing staff optimise the performance of fast track systems as they have the ability to make timely treatment and disposition decisions with minimal consultation. ${ }^{1489}$ In addition, dedicated fast track staffing is thought to promote faster patient throughput by reducing handovers and fragmentation of ED care. ${ }^{14} 8$
Although $83.4 \%$ of Victorian ED roster specific staff to their fast track area, there is variation in the staffing profile of fast track systems across Victoria. ${ }^{7}$ The majority of ED used a combination of medical and nursing staff; however, $86.7 \%$ of $\mathrm{ED}$ with dedicated fast track staffing allocated staff using a rotating roster. ${ }^{7}$ The level of medical staff rostered to fast tracks systems was variable and ranged from emergency physicians to interns. ${ }^{7}$ In contrast, the majority of ED used senior nursing staff in their fast track areas and $24.5 \%$ of nurses working in fast track areas across Victoria are emergency nurse practitioners/candidates. A small number of ED employed allied health clinicians in their fast track services, including physiotherapists, occupational therapists and care coordinators. ${ }^{7}$

Although there are a number of benefits associated with ED fast track, including decreased waiting time, ${ }^{10}$ shorter ED LOS, ${ }^{3} 5^{11-16}$ and reduced left-before-treatment rates, ${ }^{10}{ }^{16}$ little is known about how clinician designation affects fast track performance. Despite the rapid growth of fast track systems in Australia, the impact of various staffing profiles is poorly understood, and the presumed benefits of using senior staff are based on theoretical suppositions. The aim of this audit was to evaluate the effect of clinician designation on ED fast track performance.

\section{METHODS}

\section{Study design}

A retrospective audit of all patients managed by the fast track system in a metropolitan ED during 2008. This audit was approved by the organisational quality committee who deemed the audit met the National Health and Medical Research Council criteria for a quality assurance activity. ${ }^{17}$ Submission of a full application to the Human Research and Ethics Committee was waived.

\section{Setting}

The study setting was the Northern Hospital, Northern Health (TNH), a 300-bed metropolitan teaching hospital in Melbourne, Australia. The ED at TNH provided care for 61093 patients during 2008. Children aged less than 16 years comprised $20 \%(n=12237)$ of presentations and the admission rate was $25 \%$ including short stay unit and medical assessment and planning unit admissions.

Fast track was implemented in ED at $\mathrm{TNH}$ in November 2006 and operates from 10:00 to 02:00 hours to manage peak presentation times. Fast track at TNH is currently focused on the management of patients with specific non-urgent complaints who are expected to be discharged from the $\mathrm{ED}$ and do not require trolley care, intravenous 
analgesia or intravenous fluids. Patients suitable for management under the fast track model of care are identified by the triage nurse using specific inclusion criteria based on presenting problems irrespective of triage category. Presenting problems that may be managed in fast track include: localised cellulitis, wounds and lacerations, epistaxis, foreign bodies, insect bites, minor burns, minor eye complaints, body fluid exposures, single distal limb injuries. Patients are allocated directly to fast track by the triage nurse and are not managed by any of the other ED systems aimed at expediting the care of patients in the waiting room.

Fast track has dedicated staffing and fast track staff should not be responsible for patient care or the supervision of less experienced medical and nursing staff in other areas of the ED. Ideal staffing is at an ED registrar, emergency physician or emergency nurse practitioner/candidate supported by a senior emergency nurse. In Victoria, emergency nurse practitioners are authorised to manage specific patient groups independently including assessment, ordering and interpreting diagnostics, prescribing medication, discharge and referral to specialists. Emergency nurse practitioner candidate refers to nurses who are working towards endorsement as an emergency nurse practitioner. In the context of managing patients in fast track, the scope of practice of emergency nurse practitioners/candidates is interchangeable with that of ED medical staff and emergency nurse practitioners/candidates are part of the medical roster. The emergency nurses rostered to fast track are competent in the triage role indicating capacity for advanced clinical decisionmaking. They also have specific educational preparation in the following advanced clinical skills: management of distal limb injuries (including initiating diagnostic imaging and simple plaster of Paris application); management of lacerations and wounds (including use of wound adhesives); and management of plaster of Paris complications.

\section{Participants}

A de-identified dataset of all patients triaged to ED fast track from 1 January 2008 to 31 December 2008 was provided by the health information service. The dataset comprised the following fields: patient age; time and date of $\mathrm{ED}$ presentation; triage category; ED LOS; treating clinician and discharge destination.

\section{Data analysis}

The main outcome measures for this audit were: waiting times per triage category with a focus on meeting Australasian triage scale (ATS) recommendations ${ }^{18} 19$ and ED LOS for non-admitted patients with a specific focus on compliance with the Victorian Department of Human Services target of $4 \mathrm{~h}$ ED LOS for at least $80 \%$ of non-admitted patients. ${ }^{20} \mathrm{ED}$ waiting time was defined as the difference between the arrival time and the time seen by a medical officer or emergency nurse practitioner. ED LOS was defined as the difference between arrival time and departure time. $^{21}$

Treating clinicians were coded as intern, junior medical officer, senior medical officer, registrar, emergency physician, emergency nurse practitioner and locum medical officer according to their designation on the ED roster. Difference in waiting times and ED LOS were examined for each clinician group. Descriptive statistics were used to summarise the audit data and when data were not normally distributed, medians and interquartile ranges (IOR) are presented. Relationships between non-parametric variables were examined using $\chi^{2}$ and the Kruskal-Wallis test was used to compare median values. Data were analysed using SPSS version 15 .

\section{RESULTS}

\section{Patient characteristics}

A total of 8714 patients was triaged to ED fast track from 1 January 2008 to 31 December 2008: patients managed in fast track therefore comprised $14.3 \%$ of total ED presentations. The median number of patients treated by fast track per day was 24 (IOR 19-28). The median patient age was 30 years (IOR 17-46) and $21 \%$ of patients $(n=1825)$ were children aged less than 16 years. The distribution of triage categories was as follows: $0.5 \%$ ATS $2(n=45), 7.5 \%$ ATS $3 \quad(n=655), 78.3 \%$ ATS 4 $(n=6819)$ and $13.7 \%$ ATS $5(n=1195)$. The median waiting time for all fast track patients was 50 min (IOR 23-96) and overall $54.4 \%$ of patients $(n=4744)$ were seen within the maximum time frame recommended by the ATS. The majority of fast track patients were discharged from the ED ( $n=7316,84 \%$ ). Admission (including short stay unit and medical assessment and planning unit) occurred in 647 patients, 26 patients were transferred to other hospitals, 191 patients left after clinical advice, 489 patients left before treatment commenced and 45 patients left against advice after treatment had commenced.

\section{Clinician characteristics}

There was variability in the designation of treating clinicians working in fast track (table 1). In total, there were 34 interns, 45 junior medical officers, 38 senior medical officers, 19 registrars, 16 emergency physicians, one emergency nurse practitioner and one emergency nurse practitioner candidate. The number of locum medical officers was unknown as they all use the same code in the ED information system. The median number of clinicians working in fast track per day was four (IOR 3-5) (table 1). There were no clinically significant differences in the triage categories of patients managed by each clinician group (table 2).

\section{Waiting time}

Overall, $54.4 \%$ of patients were seen within the time recommended by their ATS category. The percentage of patients seen within ATS recommendations by clinician group is shown in table 3. Compliance with ATS waiting time recommendations was highest for emergency nurse practitioners/candidates $(82.5 \%)$ and lowest for junior medical officers (48.2\%).

\section{ED LENGTH OF STAY}

Median ED LOS stay was $2.35 \mathrm{~h}$ (IOR 1.53-3.35) for discharged patients and $5.05 \mathrm{~h}$ (IOR 3.63-6.80) for patients requiring admission. Patients managed by emergency nurse practitioners/ candidates and emergency physicians had a significantly shorter

Table 1 Treating clinicians in ED fast track

\begin{tabular}{lcr}
\hline & \multicolumn{2}{c}{$\begin{array}{c}\text { Total patients managed } \\
\text { during study period }\end{array}$} \\
\cline { 2 - 3 } Clinician group & $\mathbf{n}$ & $\%$ \\
\hline Senior medical officer $(\mathrm{n}=38)$ & 1781 & 25.0 \\
Junior medical officer $(\mathrm{n}=45)$ & 1586 & 20.4 \\
Registrar $(\mathrm{n}=19)$ & 1190 & 18.2 \\
Nurse practitioner/nurse practitioner & & 13.7 \\
candidate $(\mathrm{n}=2)$ & 740 & 8.5 \\
Emergency physician $(\mathrm{n}=16)$ & 696 & 8.0 \\
N/A* & 492 & 5.6 \\
Intern ( $n=34)$ & 48 & 0.6 \\
Locum medical officer & 8714 & 100.0 \\
&
\end{tabular}

*Left before treatment $(n=482)$; left after advice $(n=191)$; clinician not recorded $(n=14)$; seen by medical staff from outside emergency department (ED) $(n=9)$. 
Table 2 ATS categories of ED fast track patients by treating clinician

\begin{tabular}{|c|c|c|c|c|c|c|c|c|}
\hline \multirow[b]{2}{*}{ Clinician group } & \multicolumn{2}{|c|}{ ATS 2} & \multicolumn{2}{|c|}{ ATS 3} & \multicolumn{2}{|c|}{ ATS 4} & \multicolumn{2}{|c|}{ ATS 5} \\
\hline & $\mathrm{n}$ & $\%$ & n & $\%$ & $\mathrm{n}$ & $\%$ & n & $\%$ \\
\hline Registrar & 14 & 0.9 & 124 & 7.8 & 1258 & 79.3 & 190 & 12.0 \\
\hline $\begin{array}{l}\text { Nurse practitioner/nurse practitioner } \\
\text { candidate }\end{array}$ & 7 & 0.6 & 62 & 5.2 & 949 & 79.7 & 172 & 14.5 \\
\hline Junior medical officer & 9 & 0.5 & 155 & 8.7 & 1452 & 81.5 & 165 & 9.3 \\
\hline Senior medical officer & 11 & 0.5 & 188 & 8.6 & 1700 & 77.9 & 282 & 12.9 \\
\hline Emergency physician & 3 & 0.4 & 69 & 9.3 & 554 & 74.9 & 114 & 15.4 \\
\hline$N / A^{*}$ & 0 & 0.0 & 13 & 1.9 & 485 & 69.7 & 198 & 28.4 \\
\hline
\end{tabular}

*Left before treatment $(n=482)$; left after advice $(n=191)$; clinician not recorded $(n=14)$; seen by medical staff from outside emergency department (ED) ( $n=9)$.

ATS, Australasian triage scale.

ED LOS, whereas patients managed by junior medical officers and interns had significantly longer ED LOS (table 4) $\left(\chi^{2}=498.539, \mathrm{df}=6, \mathrm{p}<0.001\right)$.

Overall, $85.8 \%$ of non-admitted fast track patients $(n=6278)$ left the ED within $4 \mathrm{~h}$. The percentage of non-admitted patients with an ED LOS less than $4 \mathrm{~h}$ by clinician group is shown in table 5 . The $80 \%$ LOS target was met by all levels of clinician except junior medical officers and interns who were very close to achieving this key performance indicator. Over $90 \%$ of patients managed by emergency nurse practitioners/candidates, emergency physicians and locum medical officers left the ED in less than $4 \mathrm{~h}$.

\section{DISCUSSION}

Research literature to date suggests that dedicated senior staff and patient selection are key factors in the success of ED fast track systems. ${ }^{1489}$ The results of this study show variability in compliance with ATS waiting time recommendations, ED LOS for non-dmitted patients and compliance with $4 \mathrm{~h}$ ED LOS target for non-dmitted patients.

The variability in results presented in this paper may have a number of possible explanations. First, variability in practice may be related to skills, knowledge and decision-aking of individuals or clinician groups. Less experienced clinicians or clinicians unfamiliar with the ED environment may take longer to assess and treat patients or require advice from more senior clinicians. It may be proposed that junior clinicians are more likely to interrupt the care of fast track patients to seek clarification or advice from more senior colleagues; however, further research incorporating clinical practice observation is warranted to confirm or refute this assumption. Second, the level of involvement that different clinician groups have with other ED patients is unclear and diversion of staff from fast track to other activities may be a confounding factor in the study results.

Table 3 Proportion of patients seen within ATS recommendations by clinician group

\begin{tabular}{lllr}
\hline & $\mathbf{n}$ & $\%$ & p Value \\
\hline $\begin{array}{l}\text { Nurse practitioner/nurse practitioner } \\
\text { candidate }\end{array}$ & $982 / 1190$ & 82.5 & $<0.001$ \\
Locum medical officer & $31 / 48$ & 64.6 & 0.430 \\
Emergency physician & $444 / 740$ & 60.0 & 0.564 \\
Intern & $287 / 492$ & 58.5 & 0.797 \\
Senior medical officer & $1258 / 2181$ & 57.7 & 0.141 \\
Registrar & $868 / 1586$ & 54.7 & $<0.001$ \\
Junior medical officer & $858 / 1781$ & 48.2 & $<0.001$ \\
\hline${ }^{*} \chi^{2}$. & & & \\
ATS, Australasian triage scale. & & &
\end{tabular}

Emergency physicians may be interrupted more than other clinician groups given the seniority of their role and expectations that they will supervise less experienced medical staff and assist in the management of complicated patients. Furthermore, emergency nurse practitioners/candidates may have lower levels of interruptions as their scope of practice guidelines and patient types managed in fast track have a natural synergy. Although medical officers and emergency nurse practitioners/candidates are rostered exclusively to fast track, the degree to which they are called on to engage in the care of other patients warrants further investigation.

Compliance with ATS waiting time recommendations was highest for emergency nurse practitioners/candidates and lowest for junior medical officers. The median ED LOS for nonadmitted patients was less than $3 \mathrm{~h}$ for all clinician groups. This finding adds to the results of other studies that show ED fast track has a positive effect on ED LOS. ${ }^{3} 5$ 11-16 The overall proportion of non-admitted patients discharged in less than $4 \mathrm{~h}$ was $78 \%$, just below the $80 \%$ target set by the Victorian Department of Human Services. ${ }^{20}$ Compliance with this performance indicator was highest for emergency nurse practitioners/candidates who met this target $95.9 \%$ of the time and lowest for interns who met this target $78.9 \%$ of the time. There were no clinically significant differences in ATS categories of patients managed by different clinician groups, so it is unlikely that patient characteristics influenced this finding. Given that emergency nurse practitioners/candidates are experienced emergency nurses, their many years of triage experience and intimate understanding of triage and waiting room management may be one possible explanation for these results. Further research into the timing and types of decisions by each clinician group in terms of assessment, ordering diagnostics and patient disposition may further explain these results.

Although the ED in this study had clear inclusion criteria for patients managed in fast track, there is variation between ED in the fast track patient selection criteria. Some ED target patients

Table 4 Median ED LOS for non-admitted patients by clinician group (h)

\begin{tabular}{lrll}
\hline & $\mathbf{n}$ & Median & IQR \\
\hline $\begin{array}{l}\text { Nurse practitioner/nurse practitioner } \\
\text { candidate }\end{array}$ & 1115 & 1.7 & $1.2-2.4$ \\
Emergency physician & 660 & 2.1 & $1.3-3.1$ \\
Registrar & 1448 & 2.4 & $1.5-3.3$ \\
Senior medical officer & 2001 & 2.4 & $1.6-3.4$ \\
Intern & 437 & 2.5 & $1.6-3.8$ \\
Locum medical officer & 40 & 2.7 & $1.6-3.3$ \\
Junior medical officer & 1592 & 2.7 & $2.0-3.8$ \\
\hline
\end{tabular}

$E D$, emergency department; IQR, interquartile range; LOS, length of stay. 
Table 5 Proportion of non-admitted patients with ED LOS of $4 \mathrm{~h}$ or less by clinician group

\begin{tabular}{lllr}
\hline & $\mathbf{n}$ & $\%$ & p Value* \\
\hline $\begin{array}{l}\text { Nurse practitioner/nurse practitioner } \\
\text { candidate }\end{array}$ & $1069 / 1115$ & 95.9 & $<0.001$ \\
Emergency physician & $594 / 660$ & 90.0 & 0.001 \\
Locum medical officer & $36 / 40$ & 90.0 & 0.447 \\
Registrar & $1262 / 1448$ & 87.2 & 0.102 \\
Senior medical officer & $1690 / 2001$ & 84.5 & 0.042 \\
Junior medical officer & $1262 / 1592$ & 79.3 & $<0.001$ \\
Intern & $345 / 437$ & 78.9 & $<0.001$ \\
\hline${ }^{*} \chi^{2}$. & & & \\
ED, emergency department; LOS, length of stay. & & &
\end{tabular}

likely to go home $e^{52}$ and other ED use case complexity rather than probable disposition as a basis for fast track inclusion criteria ${ }^{9}$ Nevertheless, the intent is the same: fast track is aimed at the management of patients with apparent diagnoses who can be managed rapidly and do not require concentrated emergency nursing care.

Recommendations that senior staff should be used in fast track are based on assumptions that increased experience and knowledge will result in logistical advantages such as increased procedural competence and speed, improved communication and rapid identification of the seriously ill patient. In this study, $5.6 \%$ of fast track patients were managed by interns and $20.4 \%$ were managed by junior medical officers. The allocation of interns and junior medical officers to fast track does not allow for the most efficient processing of large number of patients. However, it may also be argued that rostering junior medical officers and interns to fast track provides valuable training and education opportunities.

Although junior medical staff are encouraged to consult with more senior colleagues for clinical advice to ensure a high standard of safe emergency care, the need for consultation can result in significant delays in treatment and/or disposition decisions, ${ }^{6}{ }^{23}$ which is contradictory to the rapid throughput aim that underpins fast track systems. The unresolved tension of balancing the need for experience and training in the management of a variety of patient groups and the throughput aims of fast track systems remains a challenge for both emergency medicine and emergency nursing. The future development of systems to manage emergency demand needs to ensure that service provision and education and training are not mutually exclusive.

The following limitations must be considered when interpreting the results of this study. First, this study collected data using retrospective audit so data reliability and consistency may be questions, particularly as the major outcome measures are largely time related and the current ED information system is reliant on clinicians to enter data (such as the time the patient was seen and discharge time). Inclusion of data from 8714 patients managed by over 154 different clinicians over a 12-month period should limit individual clinician's variance in data entry and integrity and minimise the impact of this limitation. Second, as discussed earlier in this paper, the fast track model at $\mathrm{TNH}$ has dedicated staffing (medical officers or emergency nurse practitioners/candidates supported by senior emergency nurse); the degree to which fast track staff are used in other activities is unknown and was beyond the scope of this study. Objective assessment of the degree to which fast track medical officers and emergency nurse practitioners/candidates are used in other areas of ED and the impact of these diversions on the efficacy of fast track warrants further investigation. Third, this study focused on time-based performance indicators. Although previous small studies have shown that ED fast track can improve elements of care such as analgesia administration and time to $\mathrm{x}$-ray, ${ }^{24}$ this study did not consider quality of care indicators. Finally, this study was set in one ED thus limiting the generalisability of the study findings.

\section{CONCLUSION}

Clinician designation does impact waiting times and, to a lesser extent, ED LOS for patients managed in ED fast track systems function. Patients managed by senior clinicians (emergency nurse practitioners/candidates and emergency physicians) had the shortest ED LOS and greatest compliance with the $4 \mathrm{~h}$ ED LOS target for non-admitted patients. The challenge for the future of fast track systems is to balance increasing demand for emergency care with the provision of safe and high quality emergency care and the training of less experienced medical and nursing staff in the rapid management of a spectrum of illnesses and injuries. Future prospective research should therefore focus on better understanding the relationship between clinician expertise, timebased performance measures and quality of care indicators.

Competing interests None declared.

Ethics approval The Northern Health Quality Committee deemed the audit met the National Health and Medical Research Council criteria for a quality assurance activity. Submission of a full application to the Human Research and Ethics Committee was waived.

Contributors JC conceptualised the study, obtained and analysed the study data. JC MK and HES interpreted the study data. JC drafted the manuscript and MK and HES provided critical revision. JC, MK and HES approved the final manuscript. JC takes overall responsibility for the paper.

Provenance and peer review Not commissioned; externally peer reviewed.

\section{REFERENCES}

1. Cameron JM. Managing emergency demand in public hospitals. performance audit report. No 71. Melbourne: Office of the Auditor General, 2004. http://www.audit.vic gov.au/reports_par/medph_report.pdf (accessed 13 Sept 2007).

2. Taylor D, Bennett D, Cameron $P$. A paradigm shift in the nature of care provision in emergency departments. Emerg Med J 2004;21:681-4.

3. Cooke MW, Wilson S, Pearson S. The effect of a separate stream for minor injuries on accident and emergency department waiting times. Emerg Med $\mathrm{J}$ 2002;19:28-30.

4. Cooke MW, Fisher J, Dale J, et al. Reducing attendances and waits in emergency departments: a systematic review of present innovations. Report to the national coordinating centre for NHS service delivery and organisation R \& D (NCCSDO). Coventry: Warwick Medical School, The University of Warwick, 2004. http://www. sdo.Ishtm.ac.uk/files/project/29-final-report.pdf (accessed 31 Aug 2007).

5. Considine J, Kropman M, Kelly E, et al. Effect of emergency department fast-track on emergency department length of stay: a case-control study. Emerg Med $J$ 2008;25:815-19.

6. O'Connell TJ, Bassham JE, Bishop R0, et al. Clinical process redesign for unplanned arrivals in hospitals. Med J Aust 2008;188(6 Suppl):S18-22.

7. Victorian Department of Human Services. Discussion paper. streaming care: fast track services in hospital emergency departments. Melbourne: Metropolitan Health and Aged Care Services Division, Victorian Government Department of Human Services, 2008. http://www.health.vic.gov.au/emergency/streamingcare0109.pdf (accessed 16 Feb 2009).

8. Meislin HW, Coates SA, Cyr J, et al. Fast track: urgent care within a teaching hospital. Can it work? Ann Emerg Med 1988;17:453-6.

9. Ieraci S, Digiusto E, Sonntag P, et al. Streaming by case complexity: evaluation of a model for emergency department fast track. Emerg Med Austral 2008;20:241-9.

10. Al Darrab A, Fan J, Ferandes C, et al. How does fast track affect quality of care in the emergency department. Eur J Emerg Med 2006:13:32-5.

11. Kilic YA, Agalar FA, Kunt M, et al. Prospective, double-blind, comparative fasttracking trial in an academic emergency department during a period of limited resources. Eur J Emerg Med 1998:5:403-6.

12. Fernandes $\mathbf{C M}$, Christenson JM. Use of continuous quality improvement to facilitate patient flow through the triage and fast-track areas of an emergency department. J Emerg Med 1995;13:847-55.

13. O'Brien D, Williams A, Blondell K, et al. Impact of streaming 'fast track' emergency department patients. Aust Health Rev 2006;30:525-32.

14. Combs S, Chapman R, Bushby A. Evaluation of fast track. Accid Emerg Nurs 2007; 15:40-7 
15. Sanchez M, Smally AJ, Grant RJ, et al. Effects of a fast-track area on emergency department performance. J Emerg Med 2006;31:117-20.

16. Nash K, Zachariah B, Nitschmann J, et al. Evaluation of the fast track unit of a university emergency department. J Emerg Nurs 2007;33:14-20.

17. National Health and Medical Research Council. When does quality assurance in health care require independent ethical review? Canberra: National Health and Medical Research Council, 2003. http://www.nhmrc.gov.au/ethics/human/conduct/ guidelines/ files/e46.pdf (accessed 20 Jan 2008).

18. Australasian College for Emergency Medicine. Policy document: the Australasian triage scale. Retrieved 2 June 2010 from http://www.acem.org.au/ media/policies and guidelines/P06_Aust_Triage_Scale_-NNov_200.pdf. 2000 (reviewed 2006).

19. Australasian College for Emergency Medicine. Guidelines for implementation of the Australasian triage scale in emergency departments. Retrieved 2 June 2010 from http://www.acem.org.au/media/policies and guidelines/G24 Implementation ATS. pdf. 2000 (revised 2005)
20. Victorian Department of Human Services. Your hospitals: a report on Victoria's public hospitals July 2007 to June 2008. Melbourne: Metropolitan Health and Aged Care Services Division, Victorian Government Department of Human Services, 2008. http://www.health.vic.gov.au/yourhospitals/download.htm laccessed 7 Jan 2009)

21. Australasian College for Emergency Medicine. Policy document: standard terminology. 2001. http://www.acem.org.au/media/policies_and_guidelines/ standard terminology.pdf (accessed Sept 2008).

22. Kwa P, Blake D. Fast track: has it changed patient care in the emergency department? Emerg Med Austral 2008;20:10-15.

23. Zhu JN, Weiland TJ, Taylor DM, et al. An observational study of emergency department intern activities. Med J Aust 2008:188:514-19.

24. Considine J, Kropman M, Kelly E, et al. Effect of an emergency department fast track service on quality of care indicators. Sigma theta tau 19th international nursing research congress focusing on evidence-based practice. Singapore: Suntec Convention Centre, 2008.

\section{Images in emergency medicine}

\section{Unusual cause of suffocation in an asthmatic adult patient}

A 43-year-old man with a known history of asthma, and is on daily inhalers, presented to the emergency room (ER) because of a persistent sudden onset of difficulty of swallowing associated with throat discomfort and a feeling of "suffocation" after choking while eating soup. The patient's acute coughing and suffocation sensation improved after a few hours, but he reported persistent throat discomfort and breathlessness when he inhales forcefully, induces cough or performs any activity. He took some puffs of his inhalers but did not acquire complete relief; he decided to visit the ER for evaluation. After history taking and physical exam, which was normal, a chest $x$ ray was taken, which did not reveal abnormalities; thus, an urgent flexible endoscopic evaluation was carried out. It revealed the presence of a plant leaf, "Laurel" (figure 1A,B), which was used to add flavour to the soup, lodged behind the arytenoid processes opposing the epiglottis. It possibly acted like a valve, causing closure of the upper airways during deep inspiration effort. The patient does not recall ingestion of the leaf while sipping the soup. The leaf was removed by forceps and the patient's symptoms resolved immediately. The patient was discharged on his usual inhaler medications.

Foreign body aspiration and/or retained hypopharyngeal foreign body can be a serious medical condition and sometimes needs immediate action. The condition is usually more common in older people or those who are very young. ${ }^{1}$ However, healthy adults either underestimate aspiration of foreign bodies or fail to recognise it, and may tolerate it for a long time without acute life-threatening consequences.

In this asthmatic patient, persistent respiratory complaints following forced inspiratory effort led to suspicion of foreign body aspiration, which was confirmed and managed in the ER setting by endoscopy.

\section{A El-Tarchichi, M F Yafi, A H Debek}

Internal Medicine Department, Kingdom Hospital, Riyadh, Saudi Arabia

Correspondence to Ali Hassan Debek, Internal Medicine Department, Kingdom Hospital, P0 Box 84400, Riyadh 11671, Saudi Arabia; alidebek@yahoo.com

Competing interests Authors have no competing interests to declare.

Ethics approval This study was conducted with the approval of the educational committee of the hospital.

Patient consent Obtained.

Accepted 26 September 2008

Published Online First 8 April 2010

Emerg Med J 2010;27:842. doi:10.1136/emj.2008.067157

\section{REFERENCE}

1. Baharloo F, Veyckemans F, Francis C, et al. Tracheobronchial foreign bodies: presentation and management in children and adults. Chest 1999;115:1357-62.
Figure 1
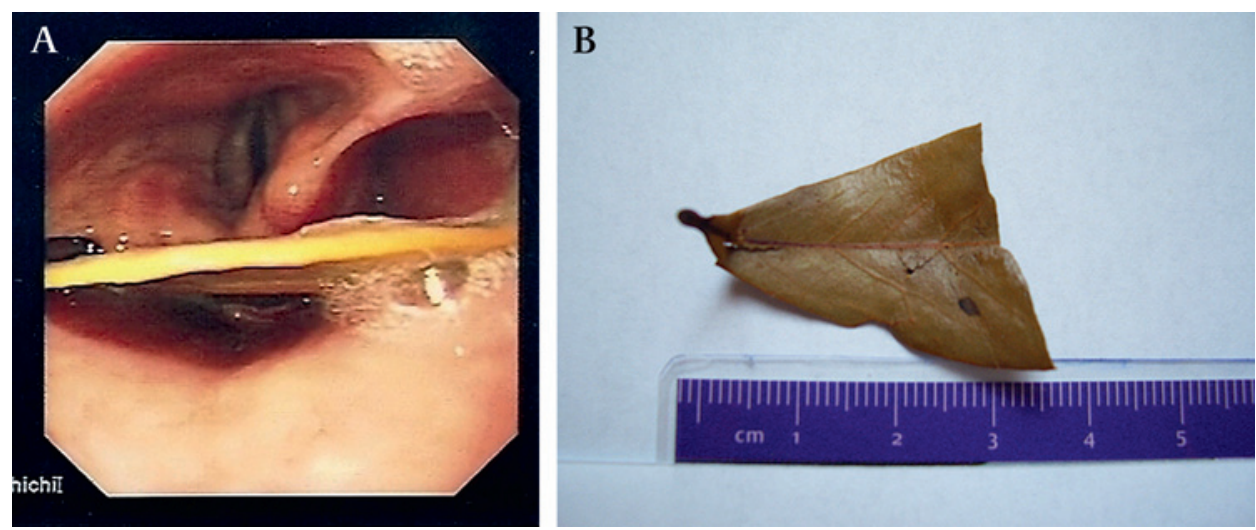